University of Nebraska - Lincoln

DigitalCommons@University of Nebraska - Lincoln

$9-1982$

\title{
Boundary Approximations for Implicit Schemes for .One- Dimensional Inviscid Equations of Gasdynamics
}

Helen Yee

R. M. Beam

R. F. Warming

Follow this and additional works at: https://digitalcommons.unl.edu/nasapub

Part of the Astrophysics and Astronomy Commons

This Article is brought to you for free and open access by the National Aeronautics and Space Administration at DigitalCommons@University of Nebraska - Lincoln. It has been accepted for inclusion in NASA Publications by an authorized administrator of DigitalCommons@University of Nebraska - Lincoln. 


\title{
Boundary Approximations for Implicit Schemes for One-Dimensional Inviscid Equations of Gasdynamics
}

\author{
H. C. Yee, ${ }^{*}$ R. M. Beam, ${ }^{*}$ and R. F. Warming $\dagger$ \\ NASA Ames Research Center, Moffett Field, Calif.
}

\begin{abstract}
The applicability to practical calculations of recent theoretical developments in the stability analysis of difference approximations is examined for initial boundary-value problems of the hyperbolic type. For the numerical experiments the one-dimensional inviscid gasdynamic equations in conservation law form are selected. A class of implicit schemes based on linear multistep methods for ordinary differential equations is chosen and the use of space or space-time extrapolations as implicit or explicit boundary schemes is emphasized. Some numerical examples with various inflow-outflow conditions highlight the commonly discussed issues: explicit vs implicit boundary schemes, and unconditionally stable schemes.
\end{abstract}

\section{Introduction}

W HEN finite-difference schemes are used to solve initial boundary-value problems for the equations of fluid dynamics, it is well known that most methods require more boundary conditions than those required by the governing partial differential equations. These additional boundary conditions for the finite-difference equations are often called "numerical boundary conditions." The numerical boundary conditions cannot be imposed arbitrarily but are determined, in general, using interior information, for example, by extrapolation or uncentered approximations. In this paper, any numerical procedure used to provide a numerical boundary condition will be called a "boundary scheme." Whatever schemes are used for the numerical boundary conditions, it is a common practice to assume that the boundary scheme has a local effect and will not affect the solution globally. During the early 1970s, Kreiss, ${ }^{1,2}$ Osher, ${ }^{3}$ Gustafsson et al., ${ }^{4}$ Varah, ${ }^{5}$ and Gustafsson ${ }^{6}$ published a series of papers establishing methods for checking the stability and accuracy of difference approximations with boundary schemes included. Since then, further progress has been made in the theory of linear difference approximations for initial boundary-value problems of the hyperbolic and parabolic type. ${ }^{7-12}$ Because improper treatment of the boundary conditions can lead to instability and inaccuracy, even though we start with a stable interior scheme (i.e., scheme for the interior points), it is appropriate to adopt an approach that includes the stability and accuracy of the combined interior and boundary schemes. Surveys of recent developments and extensive bibliographies are included in papers by Coughran ${ }^{13}$ and Yee. ${ }^{14}$

The purpose of this paper is to examine the applicability to practical calculations (for nonlinear gasdynamic problems) of recent theoretical stability analyses of implicit difference approximations for initial boundary-value problems of the hyperbolic type. As numerical computations have progressed, the use of the conservative form of the gasdynamic equations has gained popularity. For physical reasons it is sometimes desirable to specify boundary conditions in the nonconservative variables and to compute with conservative variables in the interior. We will consider the additional complications introduced by this procedure.

Presented as Paper 81-1009 at the AIAA 5th Computational Fluid Dynamics Conference, Palo Alto, Calif., June 22-23, 1981; submitted June 24, 1981; revision received Jan. 4, 1982. This paper is declared a

work of the U.S. Government and therefore is in the public domain.

${ }^{*}$ Research Scientist, Computational Fluid Dynamics Branch.

†Research Scientist, Computational Fluid Dynamics Branch. Member AIAA.
For the numerical experiments we select the onedimensional inviscid gasdynamic equations in conservation law form. We choose the class of implicit temporal schemes based on linear multistep methods ${ }^{15}$ and emphasize the use of space and space-time extrapolation as schemes for the numerical boundary conditions. These boundary schemes are the simplest and most commonly used. Through this study, we hope to gain more insight into the effect of boundary schemes on the stability of finite-difference schemes for the gasdynamic equations.

\section{Linear Stabillity Theory}

To give a background and some results of the stability theory of Kreiss ${ }^{1}$ and Gustafsson et al., ${ }^{4}$ we consider the scalar hyperbolic equation

$$
u_{t}+c u_{x}=0, \quad 0 \leq x \leq 1, \quad t \geq 0
$$

with the initial condition

$$
u(x, 0)=f(x)
$$

For a well-posed problem, ${ }^{16}$ we must specify an analytical boundary condition at the right boundary $(x=1)$ if $c$ is negative or at the left boundary $(x=0)$ if $c$ is positive. Hence, in addition to Eq. (1a) and the initial data, we specify the analytical boundary condition as either

$$
u(1, t)=g_{1}(t), \quad c<0
$$

or

$$
u(0, t)=g_{0}(t), \quad c>0
$$

If $u_{x}$ in Eq. (1a) is replaced by a three-point centered finitedifference approximation one needs a numerical boundary condition at $x=1$ if $c>0$ or at $x=0$ if $c<0$. (If a centered approximation for $u_{x}$ involves more than three points, then numerical boundary conditions would be required on both the left and right boundaries.) Therefore a procedure is needed to specify the numerical boundary conditions. Note that if we used the first-order upwind (one-sided) or second-order Padé upwind spatial difference in place of a central spatial difference, we would not need a numerical boundary condition for this problem. For systems of hyperbolic equations with mixed positive and negative eigenvalues (e.g., the gasdynamic equations) central spatial differencing is the simplest approximation to implement.

Without loss of generality we consider only the case $c<0$. Let $u_{j}^{n}$ denote the numerical approximation to $u(x, t)$ 
Table 1 Partial list of one- and two-step methods

\begin{tabular}{clllc}
\hline \hline$\theta$ & $\xi$ & $\phi$ & \multicolumn{1}{c}{ Method } & Order \\
\hline 1 & 0 & 0 & Backward Euler & 1 \\
$1 / 2$ & 0 & 0 & One-step trapezoidal & 2 \\
1 & $1 / 2$ & 0 & Backward differentiation & 2 \\
$3 / 4$ & 0 & $-1 / 4$ & Adams type & 2 \\
$1 / 3$ & $-1 / 2$ & $-1 / 3$ & Lees type & 2 \\
$1 / 2$ & $-1 / 2$ & $-1 / 2$ & Two-step trapezoidal & 2 \\
$5 / 9$ & $-1 / 6$ & $-2 / 9$ & A-contractive & 2 \\
\hline \hline
\end{tabular}

$=u(j \Delta x, n \Delta t)$ where $\Delta x$ is the spatial grid size and $\Delta t$ is the time step. Some typical boundary schemes are as follows. Space extrapolation:

$$
\begin{aligned}
& u_{0}^{n+1}=u_{1}^{n+1} \\
& u_{0}^{n+1}=2 u_{1}^{n+1}-u_{2}^{n+1}
\end{aligned}
$$

Space-time extrapolation:

$$
\begin{aligned}
& u_{0}^{n+1}=u_{1}^{n} \\
& u_{0}^{n+1}=2 u_{1}^{n}-u_{2}^{n-1} \\
& u_{0}^{n+1}=2 u_{1}^{n}-u_{2}^{n}
\end{aligned}
$$

One-sided scheme:

$$
u_{0}^{n+1}=u_{0}^{n}-c \Delta t\left(\frac{u_{1}^{n}-u_{0}^{n}}{\Delta x}\right)
$$

Box scheme:

$$
u_{0}^{n+1}+u_{1}^{n+1}+c \Delta t\left(\frac{u_{1}^{n+1}-u_{0}^{n+1}}{\Delta x}\right)=u_{0}^{n}+u_{1}^{n}-c \Delta t\left(\frac{u_{1}^{n}-u_{0}^{n}}{\Delta x}\right)
$$

In the present investigation we consider the class of interior schemes that evolves from linear multistep methods in ordinary differential equations. ${ }^{15}$ We are particularly interested in the A-stable ${ }^{17}$ (i.e., unconditionally stable) two-step methods. For the model Eq. (1a) with three-point central spatial differencing, this class of schemes is of the form

$$
\rho(E) u_{j}^{n}=-c \Delta t \sigma(E)\left(\frac{u_{j+1}^{n}-u_{j-1}^{n}}{2 \Delta x}\right)
$$

where $E$ is the shift operator defined by $E u_{j}^{n}=u_{j}^{n+1}$, and $\rho(E)$ and $\sigma(E)$ are polynomials defined by

$$
\begin{aligned}
& \rho(E)=(1+\xi) E^{2}-(1+2 \xi) E+\xi \\
& \sigma(E)=\theta E^{2}+(1-\theta+\phi) E-\phi
\end{aligned}
$$

Some well-known implicit methods belonging to this class are listed in Table $1 .{ }^{15}$ For the class of all two-step methods that are at least second-order accurate, the parameters $\theta, \xi$, and $\phi$ are related by

$$
\phi=\xi-\theta+1 / 2
$$

The two-step methods defined by Eqs. (3) are A-stable if and only if

$$
\begin{aligned}
& \theta \geq \phi+1 / 2 \\
& \xi \geq-1 / 2 \\
& \xi \leq \theta+\phi-1 / 2
\end{aligned}
$$

All methods listed in Table 1 are A-stable.
The class of second-order accurate A-stable methods is determined by Eqs. (4) and (5). In this case two parameters, $\theta$ and $\xi$, remain; that is,

$$
\xi \leq 2 \theta-1, \quad \xi \geq-1 / 2
$$

Kreiss $^{18}$ has shown that the stability of a difference ap. proximation for the initial boundary-value problem [Eqs $(1 \mathrm{a}-\mathrm{c})]$ on the interval $0 \leq x \leq 1$ is related to the stability of the difference approximation applied to the Cauchy problem

$$
\left.\begin{array}{cc}
u_{t}+c u_{x}=0, & -\infty<x<\infty, \quad t \geq 0 \\
u(x, 0)=f(x) & c<0
\end{array}\right\}
$$

and to the two related quarter-plane problems: the related right-quarter plane problem,

$$
\left.\begin{array}{cc}
u_{t}+c u_{x}=0, & 0 \leq x<\infty, \quad t \geq 0 \\
u(x, 0)=f(x) & c<0
\end{array}\right\}
$$

and the related left quarter-plane problem,

$$
\begin{aligned}
& u_{t}+c u_{x}=0, \\
& u(x, 0)=f(x) \\
& u(1, t)=g_{1}(t)
\end{aligned}
$$

Note that the numerical boundary condition associated with Eqs. (1) is transferred to the right quarter-plane problem [Eq. (8a)] for the interior scheme [Eqs. (3)]. Gustafsson et al. ${ }^{4}$ developed a stability theory for general-difference approximations to mixed initial boundary-value problems [e.g., Eqs. (8) ]. They, and others, ${ }^{4,7,13,19-22}$ have applied the theory (normal mode analysis) to some commonly used difference approximations with various boundary schemes. These analyses have included some particular methods from the class of interior schemes [Eqs. (3)] and boundary schemes [Eqs. (2)]. Prior to summarizing these results we consider some stability definitions and their implications.

For the precise stability definition used by Gustafsson, Kreiss, and Sundström (GKS), the reader should refer to their original paper. ${ }^{4}$ For the purposes of this paper we make the following definition: $A$ difference scheme for an initial boundary-value problem on a finite domain is said to be GKSstable if it is stable (by the von Neumannt method) for the Cauchy problem and stable [according to definition (3.3) of Ref. 4] for the related left and right quarter-plane problems.

The stability analyses that provide GKS-stability bounds are very useful since they are relatively simple for scalar equations and provide CFL limits that are directly applicable for many explicit schemes. They also provide a convenient method for eliminating undesirable numerical boundary condition schemes. However, they fail to provide a sufficient stability condition for some practical calculations with implicit schemes. [This is a result of the stability definition (3.3) of Ref. 4 which allows growing solutions if the mesh interval, $\Delta t$ or $\Delta x$, is not sufficiently small for a fixed value of $\lambda=|c| \Delta t / \Delta x$.] For example, if the backward Euler interior scheme [Eqs. (3) with $\theta=1, \xi=\phi=0$ ] is combined with the first-order space-time boundary scheme [Eq. (2d)] the GKSstability bound is $\lambda=\infty$, that is, the scheme can be said to be unconditionally GKS-stable. However, if a calculation is made with, for example, 20 spatial intervals and $\lambda=|c| \Delta t / \Delta x=17$, the numerical solution will grow without bound (i.e., the numerical scheme has an eigenvalue with modulus greater than unity). Therefore for many practical applications we need a more restrictive stability definition.

fFor the problems considered here the von Neumann test is necessary and sufficient for stability of the Cauchy problem. 


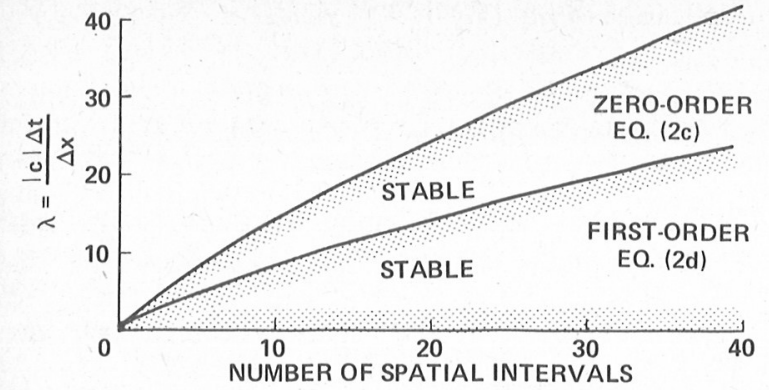

Fig. 1 P-stability bound for backward Euler and space-time extrapolation (even number of spatial intervals).

For the present discussion we will incorporate GKS-stability as a necessary condition and make the following definition: $A$ difference scheme for an initial boundary-value problem is said to be P-stable if it is GKS-stable and. all eigenvalues (corresponding to nontrivial eigensolutions) of the resolvent equations for a finite number of spatial mesh intervals have modulus less than or equal to unity.

The resolvent equations are obtained by substituting $u_{j}^{n}=z^{n} v_{j}$ into the interior scheme, boundary scheme, and the homogeneous "analytical" boundary conditions (i.e., $g_{0}(t)=0$ or $\left.g_{I}(t)=0\right)$. A detailed discussion of the analysis is presented in Ref. 23.

Here we summarize the results of various analyses for combined A-stable interior schemes and boundary schemes (2) applied to Eqs. (1):

1) All A-stable temporal interior schemes [e.g., Eqs. (3) and (5)] combined with space-extrapolation (implicit) boundary schemes [Eqs. (2a) and (2b)] are unconditionally GKS-stable and unconditionally P-stable.

2) All A-stable temporal interior schemes, [e.g., Eqs. (3) and (5)] except the "time-centered schemes" such as the trapezoidal method combined with the space-time extrapolation (explicit) boundary schemes [Eqs. (2c) and (2d)] are unconditionally GKS-stable. They are not, in general, unconditionally P-stable. For example, the backward Euler has a P-stability bound that depends on the number of spatial intervals: for an odd number of spatial intervals it is unconditionally P-stable; for an even number of spatial intervals it is conditionally P-stable with the bound a function of the number of spatial intervals (Fig. 1). The trapezoidal formula (Crank-Nicholson) is a "centered" scheme and has a GKSstability bound ${ }^{23} \lambda=|c| \Delta t / \Delta x \leq 2$.

3) Other analyses for particular A-stable interior schemes combined with boundary schemes (2e), (2f), and (2g) indicate a similar trend. For example, the backward Euler temporal scheme combined with scheme (2e) leads to a GKS- and Pstable CFL limit slightly larger than two.

We emphasize that if one combines an unconditionally stable interior scheme with a boundary scheme, the combined scheme may be unstable, conditionally stable, or unconditionally stable for the initial boundary-value problem.
These results are, of course, based on linear stability analysis of a scalar equation. Before proceeding to the numerical experiments for the nonlinear system of gasdynamic equations, we examine the results of numerical experiments for the scalar nonlinear Burgers' equation

$$
\frac{\partial u}{\partial t}+\frac{\partial\left(u^{2} / 2\right)}{\partial x}=0, \quad 0.5 \leq x \leq 1, \quad t \geq 0
$$

with initial condition

$$
u(x, 0)=x
$$

and an analytical boundary condition

$$
u(0.5, t)=0.5 /(1+t)
$$

The exact solution is

$$
u(x, t)=x /(1+t)
$$

Table 2 shows the experimental stability results when backward Euler is used for the interior scheme, and boundary schemes $(2 \mathrm{a}-2 \mathrm{~d})$ are used for the numerical boundary conditions. The experimental stability bounds correlate very closely with the linear theory results. We have also investigated the backward Euler interior scheme combined with boundary scheme (2e) applied to Burgers' equation. The experimental stability results correlate very closely with the prediction from linear theory. Gustafsson ${ }^{6}$ has proved that the numerical boundary scheme can be one order lower than the interior scheme, without reducing the global order of accuracy. The interior scheme (3a) is second-order accurate in space. The boundary schemes $(2 \mathrm{~b})$ and $(2 \mathrm{~d})$ are first-order accurate and retain second-order global accuracy is space. Boundary schemes (2a) and (2c) are zeroth-order accurate and reduce the global accuracy to first order in space. In general there is no correlation between stability and formal order of accuracy; therefore, careful treatment of numerical boundary conditions is a necessity for both accuracy and stability.

\section{One-Dimensional Gasdynamic Equations}

In one spatial dimension, the inviscid equations of gasdynamics can be written in the conservative form as

$$
\frac{\partial U}{\partial t}+\frac{\partial F(U)}{\partial x}=0
$$

where

$$
U=\left(\begin{array}{c}
\rho \\
m \\
e
\end{array}\right], \quad F(U)=\left[\begin{array}{c}
m \\
m^{2} / \rho+p \\
(e+p) m / \rho
\end{array}\right)
$$

Here $U$ is the vector of conservative variables, $F$ the flux vector, and $m=\rho u$. The primitive variables are the density $\rho$,

Table 2 Experimental maximum stable CFL number: Burgers' equation ${ }^{\mathrm{a}}$

\begin{tabular}{ccccc}
\hline \hline \multirow{2}{*}{$\begin{array}{c}\text { Number of } \\
\text { spatial } \\
\text { intervals }\end{array}$} & \multicolumn{4}{c}{ Boundary scheme ${ }^{\mathrm{b}}$} \\
\cline { 2 - 5 } & Eq. (2a) & Eq. (2b) & Eqace-time extrapolation \\
\hline \hline 19 & 380,000 & 380,000 & 380,000 & Eq. (2d) \\
\hline & $(\Delta t=10,000)$ & $(\Delta t=10,000)$ & $(\Delta t=10,000)$ & $(\Delta t=10,000)$ \\
& $($ oscil) & & $($ oscil) & 4 \\
20 & 400,000 & 400,000 & 4 & $(\Delta t=000$ \\
& $(\Delta t=10,000)$ & $(\Delta t=10,000)$ & $(\Delta t=0.1)$ & $(\Delta t=0)$ \\
& (oscil) & & $($ oscil) & \\
\hline \hline
\end{tabular}

$\mathrm{a} u(x, 0)=x ; u(0.5, t)=0.5 /(1.0+t)$. Maximum is based on sequence of $\Delta t$ sampling $(0.07,0.1$, $0.5,5,20,100,10,000)$; effective initial CFL based on $|u| \max$ at $t=0 .{ }^{\mathrm{b}}$ Applied at $x=1$. 
the velocity $u$, and the pressure $p$. The total energy per unit volume $e$ is defined as

$$
e=\rho \epsilon+\rho u^{2} / 2
$$

with $\epsilon$ as the internal energy per unit mass. The pressure $p$ for a perfect gas is defined as

$$
p=(\gamma-1)\left[e-m^{2} / 2 \rho\right]
$$

where $\gamma$ is the ratio of specific heats.

Equation (10) can be rewritten in quasilinear form

$$
\frac{\partial U}{\partial t}+A(U) \frac{\partial U}{\partial x}=0
$$

where $A$ is the Jacobian matrix $(\partial F / \partial U)$

$$
A=\left(\begin{array}{ccc}
0 & 1 & 0 \\
(\gamma-3) u^{2} / 2 & (3-\gamma) u & \gamma-1 \\
(\gamma-1) u^{3}-\gamma e u / \rho & \gamma e / \rho-3(\gamma-1) u^{2} / 2 & \gamma u
\end{array}\right]
$$

The nonlinear system of Eq. (10) can also be written in the nonconservative or primitive variable form as

$$
\frac{\partial \tilde{U}}{\partial t}+\tilde{A} \frac{\partial \tilde{U}}{\partial x}=0
$$

where

$$
\tilde{U}=\left(\begin{array}{l}
\rho \\
u \\
p
\end{array}\right], \quad \tilde{A}=\left[\begin{array}{ccc}
u & \rho & 0 \\
0 & u & 1 / \rho \\
0 & \rho c^{2} & u
\end{array}\right)
$$

and $c=(\gamma p / \rho)^{1 / 2}$ is the local speed of sound.

The matrices $A$ and $\tilde{A}$ are related by a similarity transformation

$$
\tilde{A}=M^{-1} A M
$$

with

$$
M=\left[\begin{array}{ccc}
1 & 0 & 0 \\
u & \rho & 0 \\
u^{2} / 2 & \rho u & 1 /(\gamma-1)
\end{array}\right]
$$

In addition

$$
\frac{\partial \tilde{U}}{\partial t}=M^{-1} \frac{\partial U}{\partial t}, \quad \frac{\partial \tilde{U}}{\partial x}=M^{-1} \frac{\partial U}{\partial x}
$$

The matrix $\tilde{A}$ can be diagonalized by the similarity transformation

$$
T^{-1} \tilde{A} T=\left[\begin{array}{ccc}
u & 0 & 0 \\
0 & u+c & 0 \\
0 & 0 & u-c
\end{array}\right)
$$

with

$$
T=\left(\begin{array}{ccc}
1 & \rho /(\sqrt{2} c) & \rho /(\sqrt{2} c) \\
0 & 1 / \sqrt{2} & -1 / \sqrt{2} \\
0 & \rho c / \sqrt{2} & \rho c / \sqrt{2}
\end{array}\right]
$$

Multiplication of Eq. (11) by $T^{-1}$ yields

$$
T^{-1} \frac{\partial \tilde{U}}{\partial t}+T^{-1} \tilde{A} T T^{-1} \frac{\partial \tilde{U}}{\partial x}=0
$$

If we assume that the coefficient matrices are locally constant and define

$$
W=\left[\begin{array}{l}
w_{1} \\
w_{2} \\
w_{3}
\end{array}\right]=T_{o}^{-1} \tilde{U}
$$

where the subscript $o$ is used to denote locally constant values, then we have

$$
\frac{\partial W}{\partial t}+\Lambda_{o} \frac{\partial W}{\partial x}=0
$$

where $\Lambda_{o}$ is the diagonal matrix $\left(T^{-1} \tilde{A} T\right)_{o}$ with diagonal elements $u_{o}, u_{o}+c_{o}, u_{o}-c_{o}$, and

$$
\begin{aligned}
& w_{1}=\rho-\frac{p}{c_{o}^{2}} \\
& w_{2}=\frac{1}{\sqrt{2}}\left(u+\frac{p}{\rho_{o} c_{o}}\right) \\
& w_{3}=\frac{1}{\sqrt{2}}\left(-u+\frac{p}{\rho_{o} c_{o}}\right)
\end{aligned}
$$

are the characteristic variables for the system of Eq. (11).

By adopting the notation of Beam and Warming, ${ }^{15}$ the onedimensional system of inviscid gasdynamic equations can be approximated by a linear two-step time differencing in the $\rho(E)$ form as

$$
\left(I+\omega \Delta t \frac{\partial}{\partial x} A^{n}\right) \rho(E) U^{n}=-\Delta t[\sigma(E)-\omega \rho(E)]\left(\frac{\partial F}{\partial x}\right)^{n}
$$

(Note: $\left[I+\omega \Delta t(\partial / \partial x) A^{n}\right] \rho(E) U^{n}$

$$
\left.=\rho(E) U^{n}+\omega \Delta t\left\{\partial\left[A^{n} \rho(E) U_{n}\right]\right\} / \partial x\right)
$$

The parameter $\omega=[\theta /(1+\xi)]$ is determined by the particular time-differencing approximation used (see Sec. II and Table 1). In Eq. (14) $A^{n}=A\left(U^{n}\right),(\partial F / \partial x)^{n}=\left[\partial F\left(U^{n}\right) / \partial x\right]$, and $U^{n}$ is the numerical solution at $t=n \Delta t$. with $\Delta t$ as the time step.

For linear one-step methods Eq. (14) simplifies to

$$
\left(I+\theta \Delta t \frac{\partial}{\partial x} A^{n}\right) \Delta U^{n}=-\Delta t\left(\frac{\partial F}{\partial x}\right)^{n}
$$

where

$$
\rho(E) U^{n}=\Delta U^{n}=U^{n+1}-U^{n}
$$

If $\theta=1 / 2$, the time differencing method is the trapezoidal formula and if $\theta=1$, the time-differencing method is backward Euler.

For the numerical experiments of this paper the spatial derivatives in Eq. (15) are replaced by a three-point centered difference approximation [see, e.g., Eq. (3a)]. One-sided
spatial difference approximations were considered in Ref. 14. 


\section{Numerical Boundary Conditions for the Gasdynamic Equations}

For the linear two-step time differencing in the $\rho(E)$ formulation, there are several parameters involved and there is considerable flexibility in choosing the linearized form of the numerical boundary conditions. To simplify the discussion in this section, we consider one-step methods and algorithm Eq. (15). If we denote the left and right boundary indices as 0 and $J$, the spatial differencing of Eq. (14) on the first and last computational points $(j=1, J-1)$ involves terms such as $B_{0} \Delta U_{0}^{n}, B_{J} \Delta U_{J}^{n}$ where $B_{0}, B_{J}$ are some known matrices determined from the previous time step, and $\Delta U_{0}^{n}=U_{0}^{n+1}$ $-U_{0}^{n}$. The vectors $\Delta U_{0}^{n}, \Delta U_{J}^{n}$ are partially known from the analytical boundary conditions. A few of the methods of obtaining boundary schemes are

1) Extrapolating in space or space-time. ${ }^{24,25}$

2) Discretizing the Riemann invariant equations or the characteristic equations (13) locally. ${ }^{26}$

3) Taking derivatives of the known condition in order to produce an extra boundary condition. ${ }^{9,27}$

4) Using nonreflecting boundary conditions. ${ }^{8,28,29}$

5) Overspecifying the boundary conditions.

For implicit schemes, methods 1-4 are quite complicated to implement into a computer code. Method 5 is of limited usefulness since it requires a priori knowledge of the solution at the boundary. Method 1 has the advantage of being the easiest to implement and is widely used; therefore, our study concentrates on method 1 . We emphasize that method 1 is not necessarily the optimal boundary scheme for stability, accuracy, or acceleration to steady-state solution. Our main purpose here is to try to understand more about the applicability of linear stability theory when these boundary schemes are applied to the gasdynamic equations.

From the computational point of view, it is most convenient to specify the analytical boundary data in terms of the conservative variables (if the interior scheme uses conservative variables). However, for physical reasons, one often specifies analytical boundary data in terms of the primitive variables. On the other hand, the stability analysis of scalar equations can be extended to the (linear) system if the numerical boundary extrapolations are done in the characteristic variables. ${ }^{24}$ Thus, some interesting choices of variables for the analytical boundary conditions and the numerical (or extra) boundary conditions for the conservative form [Eq. (10)] can be divided into the four groups shown in Table 3.

Under certain inflow-outflow combinations, not all of the above ways of imposing analytical boundary conditions are necessarily mathematically well posed. As an example, for a subsonic inflow with $u$ and $p$ specified (groups III and IV), the problem of Eq. (10) is not well posed.

When applicable, group I is by far the simplest to implement with the rest appearing in order of increasing complexity. Group IV, on the other hand, is more physically desirable and more theoretically sound. ${ }^{24}$ Since the numerical boundary conditions for groups II and IV are applied to the characteristic variables, the linear stability analysis can be carried out by applying the algorithm to the scalar model equation (1).

For groups I and III, the stability analysis requires dealing

with a coupled linear system that is quite complicated to

Table 3 Boundary condition specification

\begin{tabular}{lll}
\hline Group & Analytical & Numerical \\
II & B.C. variable & B.C. variable \\
II & Conservative & Conservative \\
IV & Conservative & Characteristic \\
& Primitive & Primitive \\
& Primitive & Characteristic \\
\hline
\end{tabular}

analyze. There is no complete analysis at the time of this writing, although a few cases have been analyzed by Gustafsson and Oliger. ${ }^{22}$ For the subsonic inflow case, it can be shown that all the first- and second-order A-stable approximations [Eqs. (3)] are unconditionally GKS-stable, with the following boundary conditions: $\rho_{0}^{n}$ and $u_{0}^{n}$ are given, and $p_{0}^{n}=2 p_{I}^{n}-p_{2}^{n}$. For the subsonic outflow case, again it can be shown that all the first- and second-order A-stable approximations [Eqs. (3)] are unconditionally GKS-stable, with the following boundary conditions: 1) $u_{J}^{n}$ given, $\rho_{J}^{n}=2 \rho_{J-1}^{n}$ $-\rho_{J-2}^{n}$ and $p_{J}^{n}=2 p_{J-1}^{n}-p_{J-2}^{n} ;$ or 2) $p_{J}^{n}$ given, $\rho_{J}^{n}=2 \rho_{J-1}^{n}$ $-\rho_{J-2}^{n}$ and $u_{J}^{n}=2 u_{J-1}^{n}-u_{J-2}^{n}$.

Next, we describe the space extrapolation in the characteristic variables (numerical boundary conditions) when the primitive variables are imposed as analytical boundary conditions (group IV). Other groups can follow similar procedures. We assume that the problem of Eq. (10) is well posed. A relation between the primitive and characteristic variables is

$$
T^{-1} \tilde{U}_{t}=W_{t}
$$

with $\tilde{U}$ the yector of primitive variables and $W$ the vector of characteristic variables, as defined in Eqs. (11) and (13). One of the most straightforward and compact procedures for the formulation of group IV at an inflow (left) boundary is:

1) Make a first-order approximation:

$$
\left(T^{-1}\right)_{0}^{n} \Delta \tilde{U}_{0}^{n} \approx \Delta W_{0}^{n}
$$

2) Reorder $\tilde{U}_{0}^{n}$ into subvectors $\left(\tilde{U}^{\mathrm{I}}\right)_{0}^{n}$ and $\left(\tilde{U}^{\mathrm{II}}\right)_{0}^{n}$ where $\left(\tilde{U}^{\mathrm{I}}\right)_{0}^{n}$ is the analytical boundary condition and $\left(\tilde{U}^{\mathrm{II}}\right)_{0}^{n}$ the numerical boundary condition.

3) Reorder $W_{0}^{n}$ into subvectors $\left(W^{\mathrm{I}}\right)_{0}^{n}$ and $\left(W^{\mathrm{II}}\right)_{0}^{n}$ where $\left(W^{\mathrm{I}}\right)_{0}^{n}$ corresponds to the subvector associated with the positive eigenvalues of $\tilde{A}$ and $\left(W^{\mathrm{II}}\right)_{0}^{n}$ corresponds to the negative eigenvalues of $\tilde{A}$.

4) Reorder and partition $\left(T^{-1}\right)_{0}^{n}$ as

$$
\left[\begin{array}{ll}
P_{1} & P_{2} \\
P_{3} & P_{4}
\end{array}\right]_{0}^{n}
$$

Then we have

$$
\left(\begin{array}{ll}
P_{1} & P_{2} \\
P_{3} & P_{4}
\end{array}\right]_{0}^{n}\left[\begin{array}{c}
\Delta \tilde{U}^{\mathrm{I}} \\
\Delta \tilde{U}^{\mathrm{II}}
\end{array}\right]_{0}^{n}=\left[\begin{array}{c}
\Delta W^{\mathrm{I}} \\
\Delta W^{\mathrm{II}}
\end{array}\right]_{0}^{n}
$$

Note that the delta formulation $(\Delta U)$ is important for step 1 because of the nonlinear relation between conservative, primitive, and characteristic variables.

Space extrapolation in the characteristic variables means

$$
\left(W^{\mathrm{II}}\right)_{0}^{n+1}=\alpha_{1}\left(W^{\mathrm{II}}\right)_{1}^{n+1}+\alpha_{2}\left(W^{\mathrm{II}}\right)_{2}^{n+1}
$$

with $\alpha_{1}=2, \alpha_{2}=-1$, first-order characteristic extrapolation, and $\alpha_{1}=1, \alpha_{2}=0$, zeroth-order characteristic extrapolation. This implies

$$
\begin{aligned}
& \left(P_{3} \Delta \tilde{U}^{\mathrm{I}}+P_{4} \Delta \tilde{U}^{\mathrm{II}}\right)_{0}^{n}=\alpha_{1}\left(P_{3} \Delta \tilde{U}^{\mathrm{I}}+P_{4} \Delta \tilde{U}^{\mathrm{II}}\right)_{1}^{n} \\
& \quad+\alpha_{2}\left(P_{3} \Delta \tilde{U}^{\mathrm{I}}+P_{4} \Delta \tilde{U}^{\mathrm{II}}\right)_{2}^{n}
\end{aligned}
$$

Since $P_{4}$ is nonsingular for a well-posed problem, we can rearrange terms and obtain

$$
\left(\Delta \tilde{U}^{\mathrm{II}}\right)_{0}^{n}=R_{0}\left(\Delta \tilde{U}^{\mathrm{I}}\right)_{0}^{n}+R_{1}(\Delta \tilde{U})_{1}^{n}+R_{2}(\Delta \tilde{U})_{2}^{n}
$$

where $R_{0}, R_{1}$, and $R_{2}$ are known rectangular matrices which can be evaluated from the previous time step. Similarly, the 


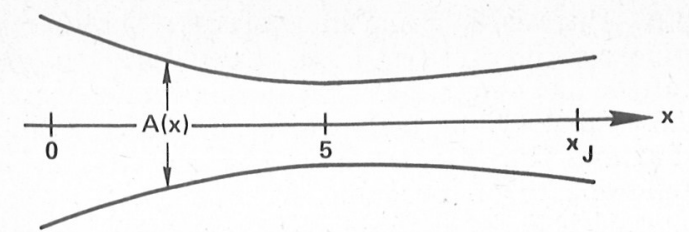

$$
\begin{array}{ll}
A(x)=1+\left(A_{0}-1\right)[(5-x) / 5]^{2} & x \leqslant 5 \\
A(x)=1+\left(A_{J}-1\right)\left[(x-5) /\left(x_{J}-5\right)\right]^{2} & x>5
\end{array}
$$

Fig. 2 Convergent-divergent nozzle ${ }^{25}\left(A_{0}=\right.$ entrance area, $A_{J}=$ exit area).

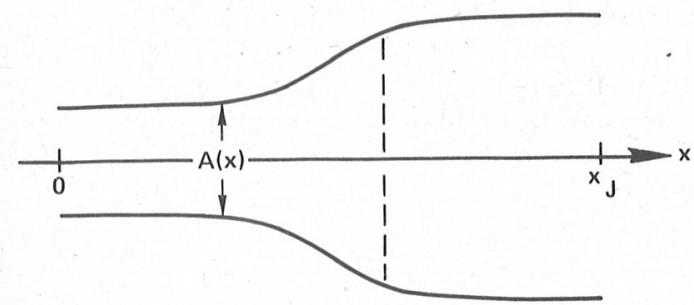

$A(x)=1.398+0.347 * \tanh (0.8 x-4)$

Fig. 3 Divergent nozzle. ${ }^{30}$

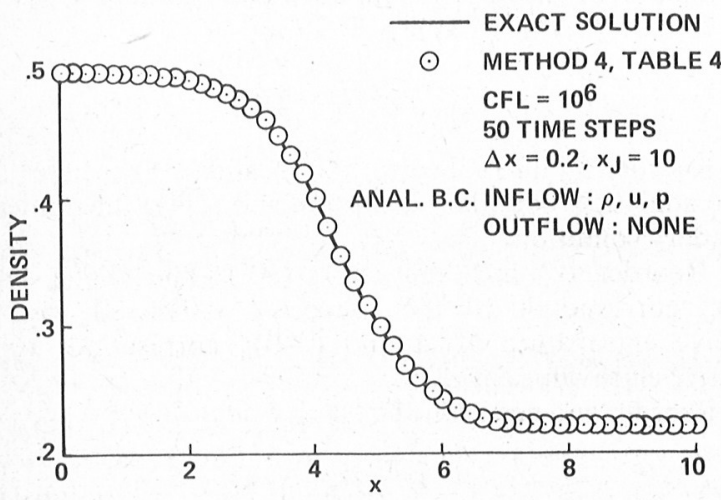

Fig. 4 Density distribution (for divergent nozzle): supersonic inflow, supersonic outflow, implicit boundary scheme (2b).

outflow numerical boundary conditions can be expressed as

$$
\left(\Delta \tilde{U}^{\mathrm{II}}\right)_{J}^{n}=S_{0}\left(\Delta \tilde{U}^{\mathrm{I}}\right)_{J}^{n}+S_{1}(\Delta \tilde{U})_{J-1}^{n}+S_{2}(\Delta \tilde{U})_{j-2}^{n}
$$

where $S_{0}, S_{1}$, and $S_{2}$ are known rectangular matrices.

Since we want to apply the interior difference approximations to the conservative form [Eq. (10)], we need to transform the values of $\Delta \tilde{U}_{j}^{n}$ at stations 1 and 2 , and $J-1$, $J-2$ appearing on the right-hand sides of Eqs. (16) and (17) into conservative variables. The method of transforming the variables is not unique since it depends on the type of linearization used. An obvious procedure is to use the linearized form

$$
\left(M^{-1}\right){ }_{j}^{n} \Delta U_{j}^{n}=(\Delta \tilde{U})_{j}^{n} \quad j=1,2, J-1, J-2
$$

where the elements of $M^{-1}$ as defined in Eq. (12) are evaluated from the previous time step. We will use this method in all of the numerical results shown in the next section. It can be shown that other methods of linearization can achieve a larger stability bound for some problems; however, this is highly problem- and scheme-dependent.

If, instead of using space extrapolation for the numerical boundary conditions, we discretize the characteristic equation and obtain an expression for $\left(\Delta \tilde{U}^{\mathrm{II}}\right)_{0}^{n}$, the counterpart of the $R_{j}$ and $S_{j}$ will be more complicated.

Two ways of implementing the implicit boundary schemes are as follows:

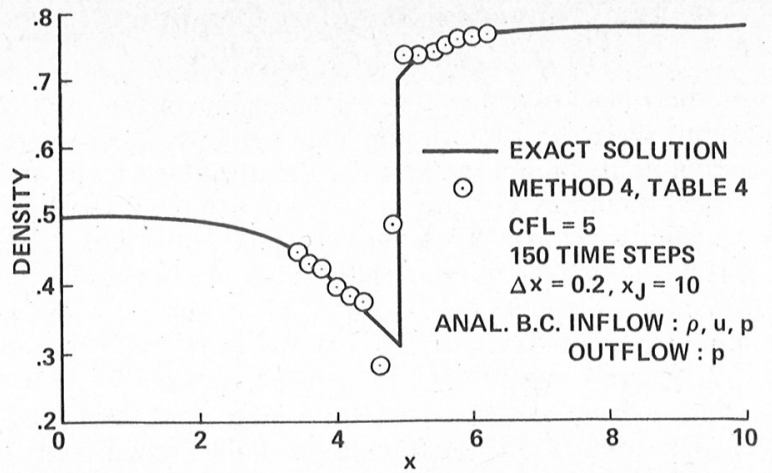

Fig. 5 Density distribution (for divergent nozzle): supersonic inflow, subsonic outflow, implicit boundary scheme ( $2 b)$.

1) Add correction matrices from, for example, Eqs. (16) and (17) to the first and last block rows of the block tridiagonal matrix.

2) Use for example, Eqs. (16) and (17) as extra equations to form the block tridiagonal matrix.

A word of caution is necessary: the final form of the matrix might not be in block tridiagonal form. But, we can use the first-order extrapolation for method 1 and the zeroth-order extrapolation for method 2 without disturbing the block tridiagonal structure. The correction matrices are (interior) scheme-independent, and thus provide a compact form for computer implementation.

\section{Numerical Results}

The stability theory for the initial boundary-value problem is based on linear theory. On the other hand, the equations of gasdynamics are nonlinear. In this section we apply the equations of gasdynamics to a quasi-one-dimensional nozzle problem and conduct numerical experiments to evaluate the applicability of linear stability theory. Implicit numerical algorithms applied to the unsteady equations are used to obtain steady-state solutions for various inflow-outflow conditions. The results of the numerical experiments are used to evaluate the effect of the numerical boundary condition on the allowable time step. We do not examine the accuracy of the transient solution.

The nozzles we consider are a convergent-divergent nozzle ${ }^{25}$ (Fig. 2) and the divergent nozzle ${ }^{30}$ (Fig. 3). We investigate five flow conditions: 1) supersonic inflow, supersonic outflow (divergent nozzle); 2) supersonic inflow, subsonic outflow (divergent nozzle); 3) subsonic inflow, supersonic outflow (convergent-divergent symmetric nozzle with entrance area $=2)$; 4) subsonic inflow, subsonic outflow without shock (convergent-divergent nozzle, area ratio $2 / 1.16$ ); and 5) subsonic inflow, subsonic outflow with shock (convergentdivergent nozzle, area ratio 2.5/1.5). Analytical and typical numerical steady-state solutions are shown in Figs. 4-8.

There are, of course, many different numerical schemes, even if we limit our choice to A-stable interior schemes and to the spatial differencing approximations of Table 4. For brevity, we choose a small class of schemes and select those for which some linear stability theory is available.

1) Temporal differencing. Of the class of A-stable methods [Eqs. (3) and (5)], the P-stability analysis is most complete for the one-step methods $(\xi=\phi=0)$ which include the trapezoidal formula and backward Euler. The GKS- and P stability bound for the trapezoidal formula with centra spatial differencing and space-time extrapolation for the numerical boundary condition is $\mathrm{CFL} \leq 2$. The trapezoida formula with central spatial differencing and space ex formula with central spatial differencing and space ap
trapolation is unconditionally GKS- and P-stable. In for plications to the gasdynamic equations the trapezoidal fo mula, which is nondissipative, did not exhibit stabili properties that were as favorable as those of the less (tem 


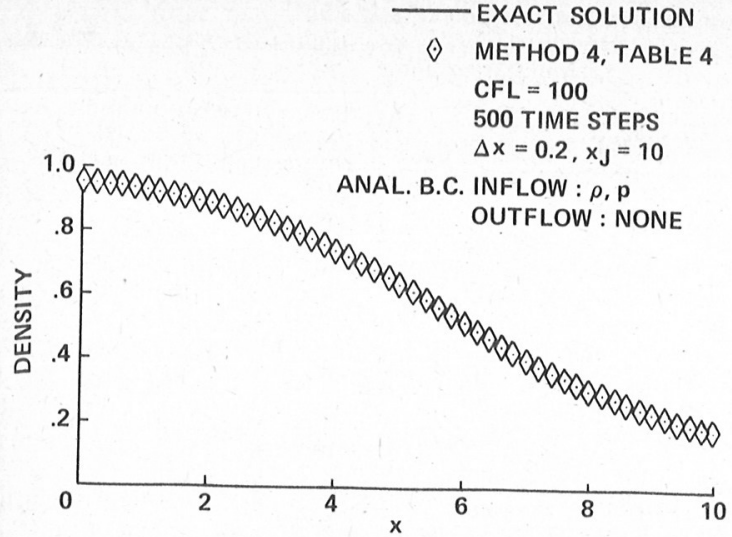

Fig. 6 Density distribution (for convergent-divergent nozzle): subsonic inflow, supersonic outflow, implicit boundary scheme (2b).

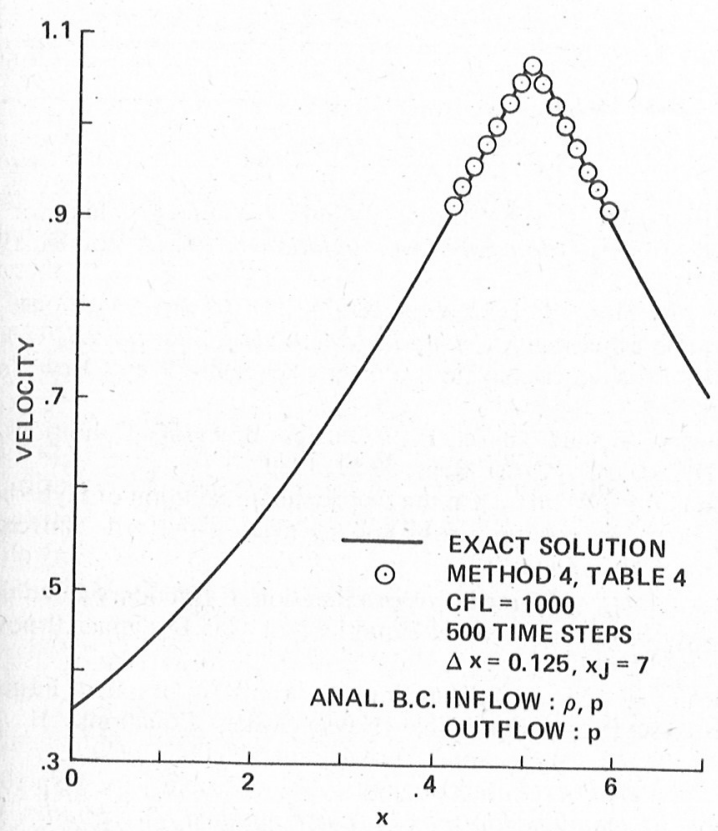

Fig. 7 Velocity distribution (for convergent-divergent nozzle): subsonic inflow, subsonic outflow, no shock, implicit boundary scheme (2b).

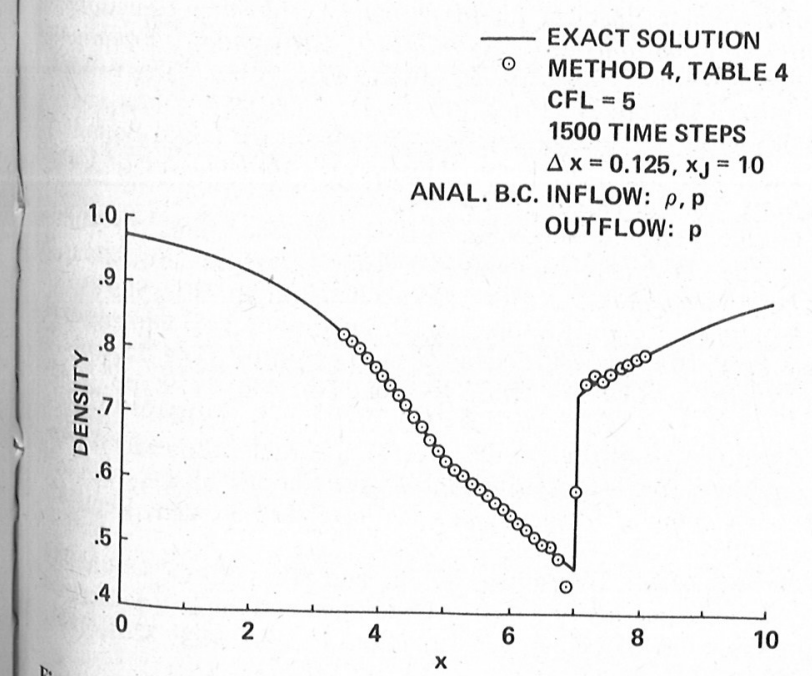

8 Density distribution (for convergent-divergent nozzle): onic inflow, subsonic outflow, with shock, implicit boundary
eme (2b).
Table 4 Spatial differencing scheme

\begin{tabular}{|c|c|c|}
\hline Method & Interior & Boundary, numerical \\
\hline 1 & $\begin{array}{l}\text { Second-order one-sided } \\
\text { (flux-vector splitting) }^{31}\end{array}$ & Eqs. (2a), (2b), (2c), (2e) \\
\hline 2 & $\begin{array}{l}\text { First-order one-sided } \\
\text { (flux-vector splitting) }^{31}\end{array}$ & Eqs. (2a), (2b), (2c), (2e) \\
\hline 3 & Central $^{\mathrm{a}, \mathrm{b}}+$ spectral norm $^{31}$ & Eqs. (2b), (2e) \\
\hline 4 & Central $^{\mathrm{a}, \mathrm{b}}$ & Eqs. $(2 a),(2 b),(2 c),(2 e)$ \\
\hline 5 & $\begin{array}{l}\text { Central }{ }^{\mathrm{a}}+\text { one-sided at } \\
\text { first and last interior } \\
\text { points }\end{array}$ & Eqs. $(2 \mathrm{a}),(2 \mathrm{~b}),(2 \mathrm{c}),(2 \mathrm{e})$ \\
\hline
\end{tabular}

${ }^{\mathrm{a}}$ Explicit fourth-order dissipation was added for the interior
scheme. ${ }^{\mathrm{b}}$ Explicit second-order dissipation was added at the first and last interior points.

poral) accurate but dissipative backward Euler. We present results for the backward Euler method.

2) Spatial differencing. In this paper, we consider only three-point central spatial-difference approximations for the interior scheme method 4 (Table 4). Other spatial difference approximations were investigated in Ref. 14. Except as noted we added an explicit fourth-order spatial dissipation term for the interior scheme and a second-order spatial dissipation term at the first and last interior points. ${ }^{15}$ The dissipation coefficient was chosen for optimum dissipation of the shortest wavelength.

3) Boundary condition variables. From the selection of boundary condition variables in Table 3 we select group IV which most closely approximates the linearized stability analysis (if we choose to specify analytical boundary conditions in terms of the primitive variables).

4) Boundary schemes. The numerical boundary conditions are treated either explicitly with either zeroth-order space-time extrapolation, as in Eq. (2c) or the modified space-time extrapolation as in Eq. (2e); or implicitly with zeroth- or firstorder space extrapolation, as in Eqs. (2a) and (2b). (Note: the variables in Eqs. (2) are now the characteristic variables.)

5) Initial conditions. In the numerical experiments we use linear interpolation between the exact steady-state boundary values as initial conditions.

The calculations were made with a series of fixed CFL numbers. Table 5 shows the maximum stable CFL number for each numerical boundary condition and each flow condition. This is based on a CFL sampling sequence $(0.5,1,1.5,2,5$, $\left.10,20,50,10^{2}, 10^{3}, 10^{6}\right)$. Typical numerical steady-state solutions are shown in Figs. 4-8 for boundary scheme (2b). Based on the results shown in Table 5 we make the following observations:

1) The implicit boundary schemes (2a) and (2b) lead to larger stability bounds than do the explicit boundary schemes (2c) and (2e).

2) For the divergent nozzle with supersonic inflow and supersonic outflow, we achieve (within machine accuracy) the unconditional linear theory stability bound for implicit boundary extrapolation. The numerical dissipation is not necessary for this flow condition.

3) For the implicit boundary schemes [e.g., (2a) and (2b)] the CFL bound is lower for flows with shocks. We conjecture that this is the result of nonlinear instability in the region of the shock. The added fourth-order dissipation is explicit and, consequently, decreases as $\Delta t$ increases, leading to insufficient dissipation in the vicinity of the shock.

4) The zeroth-order explicit and implicit boundary extrapolations [Eqs. (2a) and (2c)] lead to global accuracy problems. The solution profiles tend to be more oscillatory [than those for the first-order extrapolation, Eq. (2b)] and in one case they lead to an incorrect steady-state solution (convergent-divergent nozzle with subsonic inflow, subsonic outflow, and no shock in the exact solution). 
Table 5 Experimental maximum stable CFL number: Boundary method group IV (Table 3) ${ }^{\text {a }}$

\begin{tabular}{|c|c|c|c|c|c|}
\hline \multirow{2}{*}{ Nozzle } & \multirow{2}{*}{$\begin{array}{l}\text { Flow condition: Analytical } \\
\text { boundary condition }\end{array}$} & \multicolumn{4}{|c|}{ Boundary scheme } \\
\hline & & $\begin{array}{l}\text { Spa } \\
\text { Eq. (2a) }\end{array}$ & $\begin{array}{l}\text { ion } \\
\text { Eq. (2b) }\end{array}$ & $\begin{array}{l}\text { Space-ti } \\
\text { Eq. (2c) }\end{array}$ & $\begin{array}{l}\text { ation } \\
\text { Eq. (2e) }\end{array}$ \\
\hline Divergent & $\begin{array}{l}\text { Supersonic inflow: } \rho, u, p \\
\text { Supersonic outflow: none }\end{array}$ & $10^{6 \mathrm{~b}}$ & $10^{6 \mathrm{~b}}$ & $\begin{array}{l}10^{6 \mathrm{~b}} \text { (odd) } \\
50 \quad \text { (even) }\end{array}$ & 2 \\
\hline Divergent & $\begin{array}{l}\text { Supersonic inflow: } \rho, u, p \\
\text { Subsonic outflow: } p \\
\quad \text { (with shock) }\end{array}$ & 10 & 10 & $\begin{array}{l}5 \\
\text { (even or odd) }\end{array}$ & 1.5 \\
\hline $\begin{array}{l}\text { Convergent- } \\
\text { divergent }\end{array}$ & $\begin{array}{l}\text { Subsonic inflow: } \rho, p \\
\text { Supersonic outflow: none }\end{array}$ & $10^{2}$ & $10^{2}$ & $\begin{array}{l}5 \\
\text { (even or odd) }\end{array}$ & 2 \\
\hline $\begin{array}{l}\text { Convergent- } \\
\text { divergent }\end{array}$ & $\begin{array}{l}\text { Subsonic inflow: } \rho, p \\
\text { Subsonic out flow: } p \\
\text { (no shock) }\end{array}$ & $0.5^{\mathrm{c}}$ & $10^{3}$ & $\begin{array}{c}0.5^{\mathrm{c}} \\
\text { (even or odd) }\end{array}$ & 1 \\
\hline $\begin{array}{l}\text { Convergent- } \\
\text { divergent }\end{array}$ & $\begin{array}{l}\text { Subsonic inflow: } \rho, p \\
\text { Subsonic outflow: } p \\
\quad \text { (with shock) }\end{array}$ & 20 & 20 & $\begin{array}{l}2 \\
\text { (even or odd) }\end{array}$ & 1 \\
\hline
\end{tabular}

\footnotetext{
${ }^{\text {a }}$ Central difference in space and backward Euler in time; 49 (odd) or 50 (even) spatial intervals; maximum is based on the sequence of $\mathrm{CFL}$ sampling $(0.5,1,1.5,2,5$, $\left.10,20,50,10^{2}, 10^{3}, 10^{6}\right)$. ${ }^{b}$ Limited by machine accuracy (VAX). ${ }^{c}$ Converges to solution with shock for CFL $\geq 1$.
}

5) The linear theory for the space-time extrapolation [Eq. (2c)] predicts a stability bound which depends on the number of spatial intervals (odd number leads to better stability). This effect is seen in the computational results if the solution is smooth.

\section{Conclusion}

We have conducted numerical experiments for the onedimensional gasdynamic equations applied to nozzle flows. The numerical boundary conditions were computed implicitly (space extrapolation) or explicitly (space-time extrapolation). The experimentally determined stability bounds (maximum CFL number) correlate with the results of linearized stability theory - the implicit boundary schemes produce methods with better stability properties. The improvement is very dramatic for smooth flows but less pronounced for flows with shocks.

\section{References}

${ }^{1}$ Kreiss, H.-O., "Stability Theory for Difference Approximations of Mixed Initial Boundary Value Problems, I," Mathematics of Computations, Vol. 22, 1968, pp. 703-714.

${ }^{2}$ Kreiss, H.-O. "Difference Approximations for Initial BoundaryValue Problems," Proceedings of the Royal Society of London, Ser. $A$, Vol. 323, 1971, pp. 255-261.

${ }^{3}$ Osher, S., "Stability of Difference Approximations of Dissipative Type for Mixed Initial-Boundary Value Problems, I," Mathematics of Computations, Vol. 23, 1969, pp. 335-340.

${ }^{4}$ Gustafsson, B., Kreiss, H.-O., and Sundström, A., "Stability Theory of Difference Approximations for Mixed Initial Boundary Value Problems, II," Mathematics of Computations, Vol. 26, 1972, pp. 649-686.

5 Varah, J., "Stability of Difference Approximations to the Mixed Initial Boundary Value Problems for Parabolic Systems," SIAM Journal of Numerical Analysis, Vol. 8, 1971, pp. 598-615.

${ }^{6}$ Gustafsson, B., "The Convergence Rate for Difference Approximations to Mixed Initial Boundary Value Problems," Mathematics of Computations, Vol. 29, 1975, pp. 396-406.

${ }^{7}$ Sköllermo, G., "How the Boundary Conditions Affect the Stability and Accuracy of Some Implicit Methods for Hyperbolic Equations," Dept. of Computer Science, Uppsala University, Sweden, Rept. 62, 1975.

${ }^{8}$ Gustafsson, B. and Kreiss, H.-O., "Boundary Conditions for Time Dependent Problems with an Artificial Boundary," Journal of Computational Physics, Vol. 30, 1979, pp. 333-351.

${ }^{9}$ Gustafsson, B., "The Convergence Rate for Difference Approximations to General Mixed Initial Boundary Value Problems," Dept. of Computer Science, Uppsala University, Sweden, Rept. 85, Oct. 1979.
${ }^{10}$ Strikwerda, J. C., "Initial Boundary Value Problem for the Method of Lines," Journal of Computational Physics, Vol. 34, 1980, pp. 94-107.

${ }^{11}$ Oliger, J., "Constructing Stable Difference Methods for Hyperbolic Equations," Numerical Methods for Partial Differential Equations, edited by Seymour Parter, Academic Press, New York, 1980.

${ }^{12}$ Bayliss, A. and Turkel, E., "Outflow Boundary Conditions for Fluid Dynamics,” ICASE Rept. 80-21, 1980.

${ }^{13}$ Coughran, W. M., "On the Approximate Solution of Hyperbolic Initial-Boundary Value Problems," Thesis, Stanford University, Stanford, Calif., June 1980.

${ }^{14}$ Yee, H. C., "Numerical Approximation of Boundary Conditions with Applications to Inviscid Equations of Gas Dynamics," NASA TM-81265, 1981.

${ }^{15}$ Beam, R. M. and Warming, R. F., "An Implicit Factored Scheme for the Compressible Navier-Stokes Equations, II: The Numerical ODE Connection," AIAA Paper 79-1446, 1979.

${ }^{16}$ Kreiss, H.-O., "Initial Boundary Value Problem for Hyperbolic Systems," Communications of Pure and Applied Mathematics, Vol. 23, 1970, pp. 277-298.

${ }^{17}$ Dahlquist, G., "A Special Stability Problem for Linear Multistep Methods," Nordisk Tidskrift for Informations Behandling, Vol. 3, 1963 , pp. 27-43.

${ }^{18}$ Kreiss, H.-O., "Difference Approximations for the InitialBoundary Value Problem for Hyperbolic Differential Equations," Numerical Solutions of Nonlinear Differential Equations, Proceedings Adv. Symposium, Madison, Wisc., John Wiley \& Sons, New York, 1966, pp. 141-166.

${ }^{19}$ Oliger, J., "Hybrid Difference Methods for the Initial Boundary Value Problem for Hyperbolic Equations," Mathematics of Computations, Vol. 30, 1976, pp. 724-738.

${ }^{20}$ Gottlieb, D. and Turkel, E., "Boundary Conditions for Multistep Finite-Difference Methods for Time-Dependent Equations," Journal of Computational Physics, Vol. 26, 1978, pp. 181-196.

${ }^{21}$ Abarbanel, S. and Gottlieb, D., "Stability of Two Dimensional Initial Boundary Value Problems Using Leap-Frog Type Schemes," Mathematics of Computations, Vol. 33, No. 148, 1979, pp. 11451155 .

${ }^{22}$ Gustafsson, B. and Oliger, J., "Stable Boundary Ap." proximations for a Class of Time Discretizations of $u_{t}=A D_{o} u$," Dropt. of Computer Science, Uppsala University, Sweden, Rept. 87, Sept. 1980.

${ }^{23}$ Beam, R. M., Warming, R. F., and Yee, H. C., "Stability Analysis for Numerical Boundary Conditions and Implicit Difference Approximations of Hyperbolic Equations," NASA CP-2201, 1981, pp. 257-282.

${ }^{24}$ Gottlieb, D., Gunzburger, M., and Turkel, E., “On Numerical Boundary Treatment for Hyperbolic Systems," ICASE Rept. 78-13, Sept. 1978. 
${ }^{25}$ Griffin, M. D. and Anderson, J. D., "On the Application of Boundary Conditions to Time Dependent Computations for Quasi One-Dimensional Fluid Flows," Computers and Fluids, Vol. 5, 1977, pp. 127-137.

${ }^{26}$ Bramley, J. S. and Sloan, D. M., "A Comparison of Boundary Methods for the Numerical Solution of Hyperbolic Systems of Equations," Journal of Engineering Mathematics, Vol. 11, No. 3, 1977, pp. 227-239.

${ }^{27}$ Hyman, M., "A Method of Lines Approach to the Numerical Solution of Conservation Laws," Los Alamos Scientific Laboratory, Rept. LA-UR 79-837, 1979.

${ }^{28}$ Hedstrom, G., "Nonreflecting Boundary Conditions for Nonlinear Hyperbolic Systems," Journal of Computational Physics, Vol. 30, 1979, pp. 222-237.
${ }^{29}$ Rudy, D. and Strikwerda, J. C., "A Nonreflecting Outflow Boundary Condition for Subsonic Navier-Stokes Calculations," Journal of Computational Physics, Vol. 36, 1980, pp. 55-70.

${ }^{30}$ Shubin, G. R., Stephens, A. B., and Glaz, H. M., "Steady Shock Tracking and Newton's Method Applied to One-Dimensional Duct Flow," Journal of Computational Physics, Vol. 39, 1981, pp. 364374. Applied Mathematics Branch (R44), Naval Surface Weapons Center, Silver Spring, Md., 1980.

${ }^{31}$ Steger, J. and Warming, R. F., "Flux Vector Splitting of the Inviscid Gas-Dynamic Equations with Application to Finite Difference Methods," Journal of Computational Physics, Vol. 40, 1981, pp. 263-293.

\title{
From the AIAA Progress in Astronautics and Aeronautics Series...
}

\section{GASDYNAMICS OF DETONATIONS AND EXPLOSIONS- $-\mathrm{v} .75$ and COMBUSTION IN REACTIVE SYSTEMS-v. 76}

\author{
Edited by J. Ray Bowen, University of Wisconsin, \\ N. Manson, Université de Poitiers, \\ A. K. Oppenheim, University of California, \\ and R. I. Soloukhin, BSSR Academy of Sciences
}

The papers in Volumes 75 and 76 of this Series comprise, on a selective basis, the revised and edited manuscripts of the presentations made at the 7 th International Colloquium on Gasdynamics of Explosions and Reactive Systems, held in Göttingen, Germany, in August 1979. In the general field of combustion and flames, the phenomena of explosions and detonations involve some of the most complex processes ever to challenge the combustion scientist or gasdynamicist, simply for the reason that both gasdynamics and chemical reaction kinetics occur in an interactive manner in a very short time.

It has been only in the past two decades or so that research in the field of explosion phenomena has made substantial progress, largely due to advances in fast-response solid-state instrumentation for diagnostic experimentation and highcapacity electronic digital computers for carrying out complex theoretical studies. As the pace of such explosion research quickened, it became evident to research scientists on a broad international scale that it would be desirable to hold a regular series of international conferences devoted specifically to this aspect of combustion science (which might equally be called a special aspect of fluid-mechanical science). As the series continued to develop over the years, the topics included such special phenomena as liquid- and solid-phase explosions, initiation and ignition, nonequilibrium processes, turbulence effects, propagation of explosive waves, the detailed gasdynamic structure of detonation waves, and so on. These topics, as well as others, are included in the present two volumes. Volume 75, Gasdynamics of Detonations and Explosions, covers wall and confinement effects, liquid- and solid-phase phenomena, and cellular structure of detonations; Volume 76, Combustion in Reactive Systems, covers nonequilibrium processes, ignition, turbulence, propagation phenomena, and detailed kinetic modeling. The two volumes are recommended to the attention not only of combustion scientists in general but also to those concerned with the evolving interdisciplinary field of reactive gasdynamics.

Volume 75-468 pp., $6 \times 9$, illus., $\$ 30.00 \mathrm{Mem} ., \$ 45.00 \mathrm{List}$ Volume 76-688 pp., $6 \times 9$, illus., $\$ 30.00 \mathrm{Mem} ., \$ 45.00 \mathrm{List}$ Set-\$60.00 Mem., $\$ 75.00$ List 